\title{
CORRECTION
}

\section{Correction to: One-Day Outdoor Photometric Stereo Using Skylight Estimation}

\author{
Jiyoung Jung ${ }^{1} \cdot$ Joon-Young Lee ${ }^{2} \cdot$ In So Kweon ${ }^{3}$
}

Published online: 28 February 2019

(c) Springer Science+Business Media, LLC, part of Springer Nature 2019

\section{Correction to: \\ International Journal of Computer Vision (2019) \\ https://doi.org/10.1007/s11263-018-01145-1}

The original version of this article unfortunately contained mistakes in the figure captions. The correction information is given below:

The caption of Fig. 15 should be Fig. 16.

The caption of Fig. 16 should be Fig. 17.

The caption of Fig. 17 should be Fig. 18.

The caption of Fig. 18 should be Fig. 15.

The original article has been corrected.

The original article can be found online at https://doi.org/10.1007/ s11263-018-01145-1.

Jiyoung Jung

jiyoung.jung@khu.ac.kr

Joon-Young Lee

jolee@adobe.com

In So Kweon

iskweon77@kaist.ac.kr

1 Department of Software Convergence, Kyung Hee

University, Yongin, Republic of Korea

2 Adobe Research, San Jose, CA, USA

3 School of Electrical Engineering, KAIST, Daejeon, Republic of Korea 